\title{
Results from the observations of Forbush decreases by the Extreme Energy Events experiment
}

\author{
M. Abbresciaa ${ }^{a b}$, C. Avanzini ${ }^{a c}$, L. Baldini ${ }^{a c}$, R. Baldini Ferroli ${ }^{a d}$, G. Batignani ${ }^{a} c$, G.

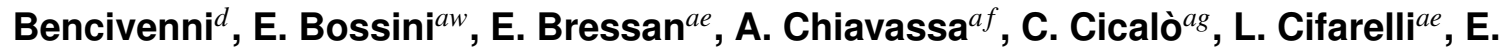 \\ Coccia $^{a h}$, A.Corvaglia ${ }^{a i}$, D. De Gruttola ${ }^{a l}$, S. De Pasquale ${ }^{a l}$, A. Di Giovanni ${ }^{m}$, M. \\ D‘Incecco $^{m}$, M. Dreucci ${ }^{d}$, F.L. Fabbri ${ }^{d}$, E. Fattibene ${ }^{n}$, A. Ferraro ${ }^{n}$, R. Forster ${ }^{o p}$, V. \\ Frolov $^{v}$, P. Galeotti ${ }^{a f}$, M. Garbini ${ }^{a e}$, G. Gemme ${ }^{q}$, I. Gnesi ${ }^{\star a f}$, S. Grazzi ${ }^{a q}$, C. \\ Gustavino $^{m}$, D. Hatzifotiadou ${ }^{a e p}$, P. La Rocca ${ }^{a r}$, A. Maggiora ${ }^{a i}$, G. Maron ${ }^{n}$, M. N. \\ Mazziotta $^{s}$, S. Miozzi ${ }^{a d h}$, F. Nozzoli ${ }^{a t}$, M. Panareo ${ }^{a i}$, M. P. Panetta ${ }^{a i}$, R. Paoletti ${ }^{a w}$, L. \\ Perasso $^{a q}$, F. Pilo ${ }^{a c}$, G. Piragino ${ }^{f}$, F. Riggi ${ }^{a r}$, G.C. Righini ${ }^{a}$, A. Rodriguez \\ Rodriguez $^{o p}$, G. Sartorelli ${ }^{a e}$, E. Scapparone ${ }^{e}$, M. Schioppa $^{a u}$, A. Scribano ${ }^{a c}$, M.

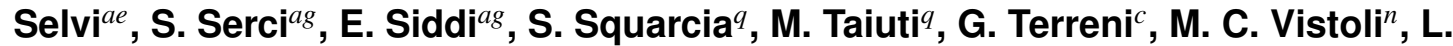 \\ Votano $^{m}$, M.C.S. Williams ${ }^{e p}$, S. Zani ${ }^{n}$, A. Zichichi ${ }^{a e p}$ and R. Zuyeuski ${ }^{o p}$ \\ a Museo Storico della Fisica e Centro Studi e Ricerche "E. Fermi", Roma, Italy \\ $b$ INFN and Dipartimento di Fisica, Università di Bari, Bari, Italy \\ c INFN and Dipartimento di Fisica, Università di Pisa, Pisa, Italy \\ d INFN Laboratori Nazionali di Frascati, Frascati (RM), Italy \\ e INFN and Dipartimento di Fisica e Astronomia, Università di Bologna, Bologna, Italy \\ $f$ INFN and Dipartimento di Fisica, Università di Torino, Torino, Italy \\ $g$ INFN and Dipartimento di Fisica, Università di Cagliari, Cagliari, Italy \\ h INFN and Dipartimento di Fisica, Università di Roma Tor Vergata, Roma, Italy \\ $i$ INFN and Dipartimento di Fisica, Università di Lecce, Lecce, Italy \\ l INFN and Dipartimento di Fisica, Università di Salerno, Salerno, Italy \\ m INFN Laboratori Nazionali del Gran Sasso, Assergi (AQ), Italy \\ $n$ INFN CNAF, Bologna, Italy \\ o ICSC World Laboratory, Geneva, Switzerland \\ p CERN, Geneva, Switzerland \\ $q$ INFN and Dipartimento di Fisica, Università di Genova, Genova, Italy \\ r INFN and Dipartimento di Fisica e Astronomia, Università di Catania, Catania, Italy \\ s INFN, Sezione di Bari, Bari, Italy \\ t INFN and ASI Science Data Center, Roma \\ u INFN and Dipartimento di Fisica, Università della Calabria, Cosenza, Italy \\ $v$ Joint Institute for Nuclear Research, Dubna, Russia \\ w INFN Gruppo Collegato di Siena and Dipartimento di Fisica, Università di Siena, Siena, Italy \\ gnesidto.infn.it
}


The EEE (Extreme Energy Event) Project is aimed to the study of Extensive Air Showers (EAS) and correlated phenomena. The experimental setup is composed by an array of more than 40 tracking telescopes, based on Multigap Resistive Plate Chambers (MRPCs) technology and distributed over a wide area covering more than $310^{5} \mathrm{Km}^{2}$. Among the different fields of investigations, the EEE telescope array is suitable for detecting galactic cosmic rays flux variations, referred therein as GCRD, mainly via the secondary muon components. Such variations are directly related to solar flares and coronal mass ejections occurring on the solar heliosphere. Four variations have already been observed in 2011, 2012, 2014 and 2015 showing the array has the capability of becoming a stable survey for GCRDs over a broad surface and more than $10^{\circ}$ in latitude and longitude.

The 34th International Cosmic Ray Conference, 30 July- 6 August, 2015

The Hague, The Netherlands

* Speaker. 


\section{Introduction}

The Extreme Energy Events [1, 2, 3] telescope array is a (growing) net of 47 tracking detectors, each made of Multigap Resistive Plate Chambers (MRPCs), spread over more than 10 degrees in latitude and 11 degrees in longitude, organized in clusters and single telescope stations. Each site hosts a muon tracking telescope made of three MRPCs, very similar, conceptually, to the chambers developed for the Time-Of-Flight system of the ALICE experiment at LHC [4, 5].

The main topics addressed by the EEE Project are related to EAS (Extensive Air Shower), i.e. short and far distance coincidences detection and also low energy phenomena such as secondary particle flux variations. However, the use of MRPCs both as tracking and timing detectors allows the reconstruction of secondary muons and electrons tracks (where the material budget above the telescope is low enough to avoid electron component to be supressed); the high time resolution of the detectors, better than $100 \mathrm{ps,}$, allows the TOF measurement of the detected particles, opening other fields, namely the study of the upward particle flux component and the angular dependence of phenomena related to secondary muon flux variations. EEE experiment performed its first regular data taking period (RUN 1) in 2015, after a Pilot Run in November 2104. A new automatic data trasmission, quality check and reconstruction running at the CNAF - IT Center for the Istituto Nazionale di Fisica Nucleare (INFN) - have been succesfully tested, making easily available the whole data set for the various analysis. Latest results overview is addressed in [6].

The EEE array is regularly growing increasing the number of clusters, with different geometries and energy threshold sensitivities.

The monitoring of Galactic Cosmic Ray flux Decreases is of interest for the understanding of phenomena that occur on the solar heliosphere, as well as on other observable stars. As it is known, they are related to the emission of mass from the star corona and often related to solar flares, even if such relation is not completely understood: several aspects still remain unclear, especially those related to the source of the phenomenon on the sun and the interplay with interplanetary structures $[7,8]$. The effect on the solar wind directly affects the measured galactic cosmic ray flux on Earth, giving typical flux fluctuations of a few percent on a few days basis. The phenomenon is therefore observable by any apparatus surveying the cosmic ray radiation with a comparable overall accuracy.

The long term survey of cosmic ray flux fluctuations has been historically performed by neutron monitors (NM), spread almost over the whole planet, and active since the last 50 years [10]. The survey experiments sensitivity to flux variations also depends on the magnetic rigidity of the secondaries, being related to modifications of the earth magnetic field. Neutron monitors show usually higher angular acceptance and lower energy threshold than EEE muon tracking detectors: this features explains the higher amplitude of the decreases when observed by NM.

On the other side the overall acceptance of each EEE station allows for a flux rate measurement within the 15-50 particle/s, depending on the set-up, while the timing within different station is driven by the GPS time resolution and it is better than $50 \mathrm{~ns}$. Therefore the EEE telescope array might allow the study of the muon component of the GCRDs as a function of the the arrival direction at different coordinates and with precise timing, giving new insights in the understanding of such phenomena. 


\section{Corrections}

The measurement of few percent secondary particle flux variations requires a set of corrections in order to cancel out systematics which may mask GCRDs. They are mainly coming from the absorption phenomena which occurs in atmosphere to the EAS shower development to the ground; this being an interaction length-related effect can be approximated with an exponential behaviour as a function of the pressure, $I=I_{0} \exp \left[-\mu\left(P-P_{0}\right)\right]$, where for small pressure variations one can consider the first order correction $\Delta I=-\mu \Delta P$. The extraction of the pressure-rate correlation for the different sites allows the correction of the pressure-induced modulation by rescaling the various measured rates to a reference pressure $P_{0}$.

Beside the corrections related to atmospheric pressure variations, MRPCs need stable internal pressure and temperature conditions in order to have uniform working conditions. These features have already been studied on large area detectors [9]. EEE sites involved in the GCRD survey are chosen among those which are installed in temperature controlled room environment, while HV correction systems are under study for a dynamic fixing of the telescopes working point.

\section{February 2011 GCRD}

The first observation of a GCRD obtained by the EEE experiment followed the large X2 solar flare of mid-February 2011. The first solar flare of the new Solar Cycle 24 happened at 1.56 UT on February 15, 2011. The event, the strongest one within the last four years, was classified as X2 on the Richter scale of solar flares, showing a maximum X-ray flux of $210^{4} \mathrm{~W} / \mathrm{m}^{2}$ near the Earth.

The measurement of the decrease was observed by 2 telescopes of the array [11], the first in Altamura (Bari) and the second one in Catania.

In order to extract reliable results, pressure corrections were applied to the telescope data. The correction factors were evaluated using pressure vs rate data correlation related to the days preceeding the GCRD.The value of the coefficients were found to be around $0.40 \% / \mathrm{mbar}$ (Altamura) and 0.28 $\% / m b a r$ (Catania). In order to minimize the rate fluctuations depending on the temperature the rooms were also kept conditioned within few degrees.

In figure 1 the fractional flux variation for the 2 EEE telescopes is plotted in comparison with the OULU NM data, showing a good agreement both in terms of amplitude and timing of the event. The decrease as measured by OULU starts around February 18th and reaches its minimum in a few hours, with an amplitude of the order of $4.5 \%$. A few hours later, the recovery phase took place, reaching nearly the original flux around February 24-25. The corresponding amplitude variation for the 2 EEE telescopes was extracted to be around 4\% (Altamura) and 3\% (Catania). The small difference in amplitude among the EEE telescopes can be explained by the different material budget above the sites, which changes their energy thresholds.

First studies on the angular dependence of the secondary particle flux during a GCRD has been performed, being expected an energy dependence of the GCRD amplitude in case of strong flares. Such dependence was not observed within the available experimental resolution.

\section{March 2012 GCRD}

A second GCRD was simultaneously observed by three EEE telescopes during March 2012 


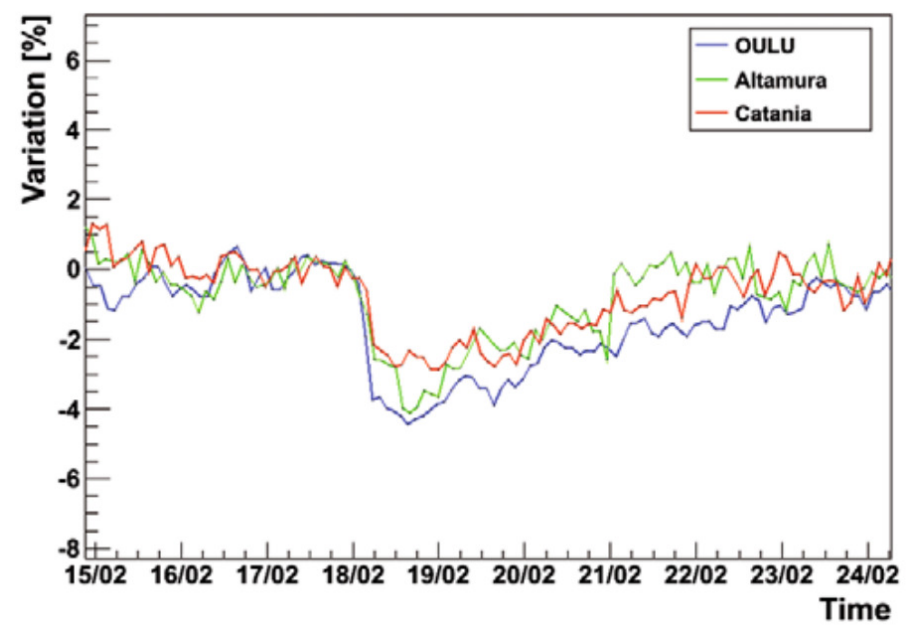

Figure 1: February 2011 flux decrease. The plot shows the flux variation as observed by 2 EEE telescopes compared with OULU neutron monitor.

[12]. This GCRD is associated to one of the largest solar flares of solar cycle 24 which happened on March 6th, categorized as an X5.4, the second largest flare since the solar activity minimum in early 2007. Figure 2 shows the flux of the secondary particles as a function of time, for the OULU $\mathrm{NM}$ (a) located in Oulu $\left(65.05^{\circ} \mathrm{N}, 25.47^{\circ} \mathrm{E}\right)$ and Rome $\mathrm{NM}\left(41.90^{\circ} \mathrm{N}, 12.52^{\circ} \mathrm{E}\right)$ and for the EEE muon telescopes $(\mathrm{b}, \mathrm{c}, \mathrm{d})$ placed in Altamura $\left(40.8^{\circ} \mathrm{N}, 16.6^{\circ} \mathrm{E}\right)$, Catania $\left(37.5^{\circ} \mathrm{N}, 15.1^{\circ} \mathrm{E}\right)$ and Bologna $\left(44.5^{\circ} \mathrm{N}, 11.3^{\circ} \mathrm{E}\right)$.

The EEE data shows a good agreement with NM data. The magnitude of the effect observed in Oulu NM w.r.t. Rome NM confirms the latitude dependence of the secondary particles flux, the GCRD being dominated by the low energy - therefore low rigidity - component of the cosmic radiation, which better reaches the ground level at lower latitudes. The same features can be observed in the case of the Bologna and Altamura EEE sites. The altitude also plays a role in the magnitude of the detected GCRD, again related to a cut of the low rigidity - low energy - secondary particles for the sites at lower altitude. Finally, the selection of the muon component operated by the EEE telescopes explains the generalized lower amplitude of the GCRD when measured by EEE sites w.r.t. NM.

\section{November 2014 GCDR}

On November 10, 2015, a flux decrease was observed at the same time by six EEE stations, covering almost the whole latitude and longitude range. It came after a X1.6 solar flare happened on November 7th at around 17.26 UT, the last of a series of medium intensity flares which happened starting on November 5th. The six telescopes which were used for the analyses of the event are summarized in table 1.

The telescope were chosen among those stable during the event; in addition they had to show clean evidence for the flux variation observation (more than $2 \sigma$ deviation w.r.t. nominal flux) and a reliable rate vs pressure correction. A typical correlation, related to SAVO-02 station, and the 


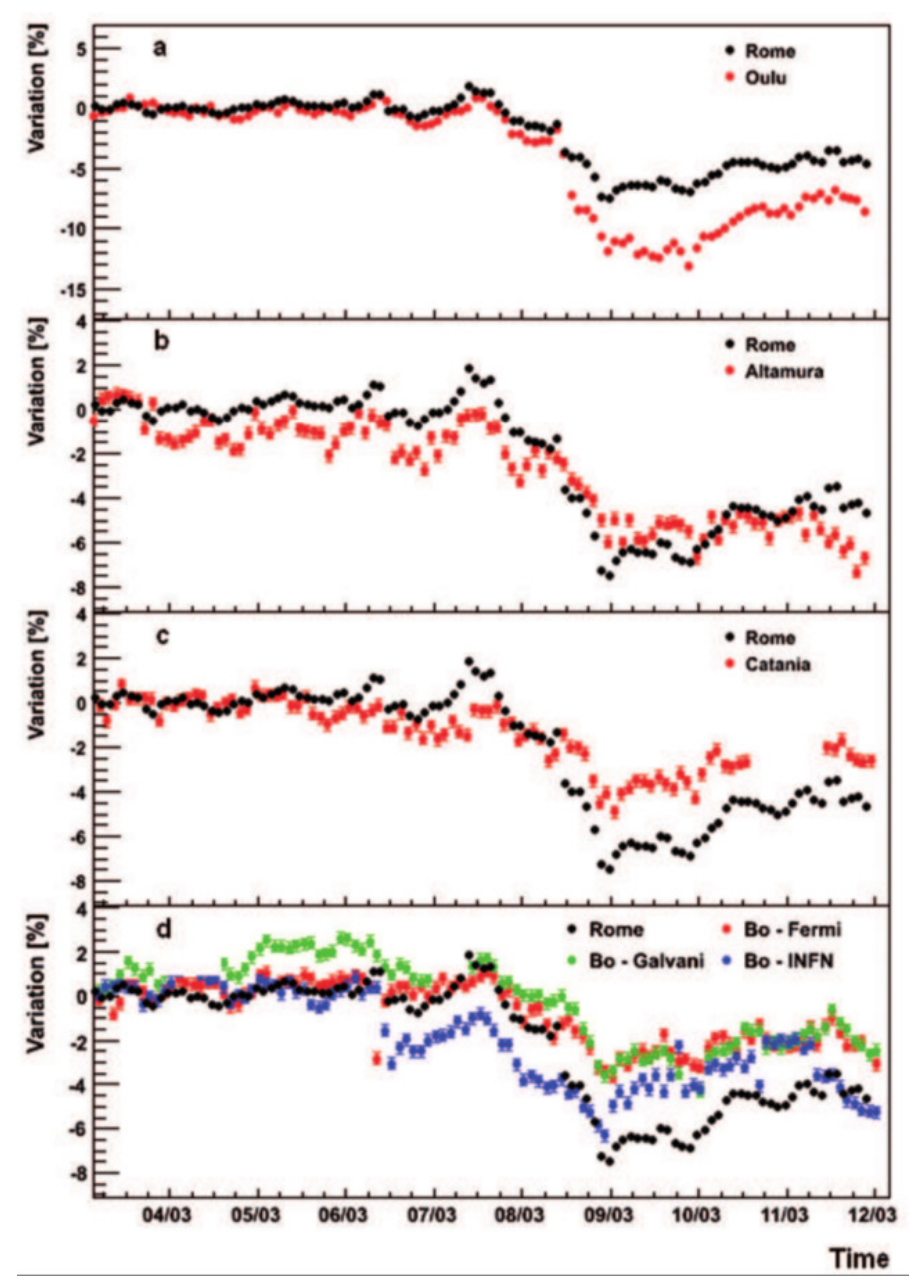

Figure 2: March 2012 flux decrease. The plot shows the flux variation as observed by Rome and Oulu NM (a) and by EEE telescopes compared to Rome NM (b,c,d).

extracted correction factor are shown in figure 3. The observation of the November 2014 GCRD

\begin{tabular}{||c|c|c|c||}
\hline \hline EEE Station & Latitude & Longitude & Altitude (m) \\
\hline ALTA-01 & $40.82^{\circ} \mathrm{N}$ & $16.55^{\circ} \mathrm{E}$ & 541 \\
\hline FRAS-02 & $41.81^{\circ} \mathrm{N}$ & $12.68^{\circ} \mathrm{E}$ & 416 \\
\hline GROS-01 & $42.75^{\circ} \mathrm{N}$ & $11.12^{\circ} \mathrm{E}$ & 20 \\
\hline SAVO-01 & $44.31^{\circ} \mathrm{N}$ & $8.48^{\circ} \mathrm{E}$ & 32 \\
\hline SAVO-02 & $44.32^{\circ} \mathrm{N}$ & $8.47^{\circ} \mathrm{E}$ & 33 \\
\hline VIAR-02 & $43.86^{\circ} \mathrm{N}$ & $10.24^{\circ} \mathrm{E}$ & 16 \\
\hline \hline
\end{tabular}

Table 1: Geographical coordinates of the telescopes used for the November 10th GCRD observation.

has been also used for testing a semi-automatic flux variation analysis chain. The chain averages the flux data over a time range, different for the various stations, in order to ensure .05 relative 


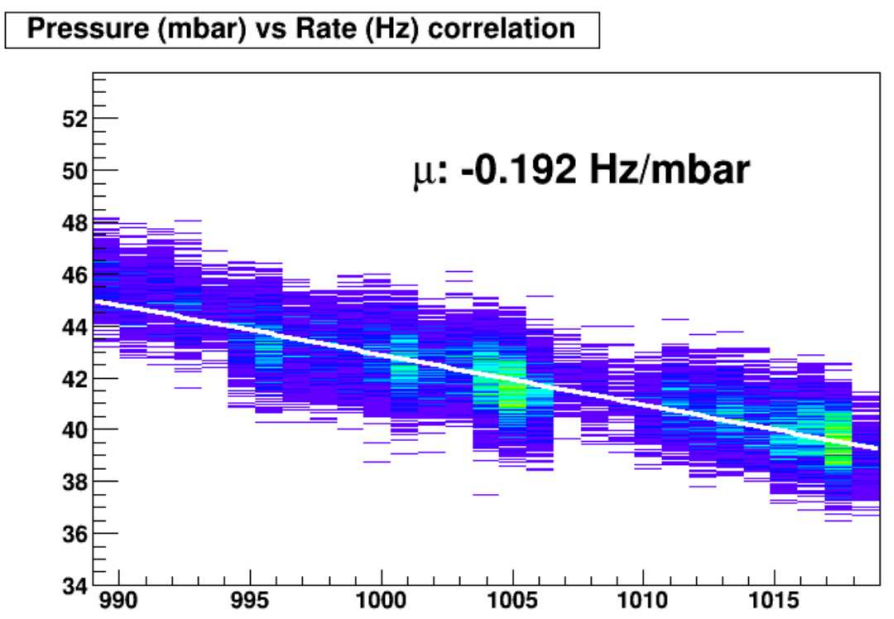

Figure 3: November 2014 GCRD. Rate vs pressure correlation and the extracted correction factor for the EEE SAVO-02 telescope.

uncertainty for any flux measurement (a typical range is 30 minutes); then a $\mathrm{N}$-point interlacing method is used for measuring the significance of the variation given in $\sigma$ units over typical time ranges of 6-12 hours. The corrections are also automatically applied where possible.

A preliminary result of the November 2014 GCRD, averaged over the six stations is shown in figure 4 in comparison with OULU neutron monitor observation, showing a good agreement. The features of the event, namely the time structure w.r.t. OULU data, and the magnitude, are under study.

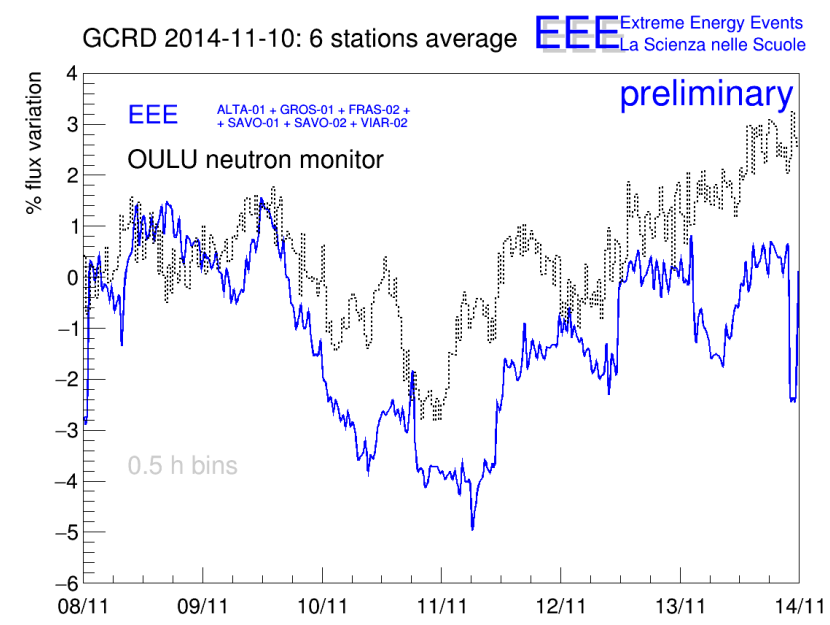

Figure 4: November 2014 flux decrease.

\section{June 2015 GCRD}

An important GCRD occured on June 23th, 2015, following a set of flares of medium magni- 
tude between June 19th and 21st.

The event was observed by 5 EEE stations, even though the array was under mantainance and upgrade. Among the 5 active telescopes we chose for the preliminary analysis CATA-02 station, which showed stable working conditions and no mantainance actions were ongoing at the site. In addition, the extraction of the pressure correction factor was performed on a set of data immediately before the GCRD.

The relative variation of the flux is showed in figure 5 in comparison with the OULU data.

Data collected by other sites are being analyzed in order to have a multi-site observation of the June 2015 GCRD: the information collected is useful for increasing the significance of the single-site measurement as well as to allow studies on latitude and altitude effects, comparisons with neutron monitor data, GCRD magnitude vs species correlations etc.

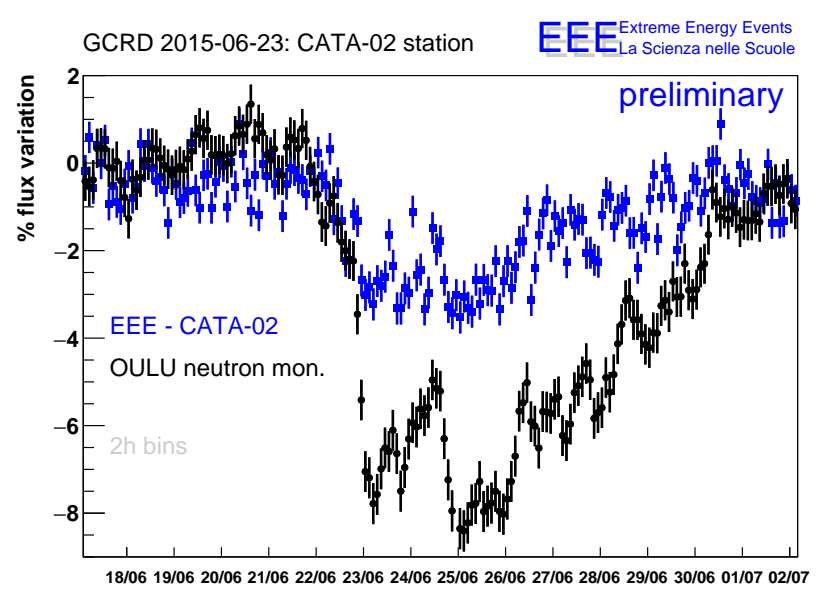

Figure 5: June 2015 flux decrease as observed by the CATA-02 station.

\section{Conclusions}

The GCRD survey through the EEE telescope array, because of its selective sensitivity to the muon component, the higher rigidity cutoff and the broad longitude/latitude and altitude coverage proves to be a complementary instrument w.r.t. to NM, allowing comparisons and the extraction of new GCRD features. These observations are promising, in view of the construction of a solar surface phenomena survey, with both high accuracy and low systematics and wide longitude/latitude coverage features.

\section{References}

[1] Centro Fermi web site: http://www.centrofermi.it/eee.

[2] A. Zichichi, Progetto "La Scienza nelle Scuole" - EEE: Extreme Energy Events (Società Italiana di Fisica, Bologna, 2004) 2nd edition (2005), 3rd edition (2012).

[3] M. Abbrescia et al., Extreme Energy Events Project: construction of the detectors and installation in Italian High Schools, Nucl. Instrum. Meth. A 588 (2008) 211. 
[4] ALICE Collaboration, Addendum to TOF Technical Design Report, CERN/LHCC, 2002-016.

[5] A. Akindinov et al., Nucl. Instrum. Methods Phys. Res. A 661, S98 (2012).

[6] M. Abbrescia et al., First results from Run1 of the Extreme Energy Events experiment, this conference proceedings.

[7] Badruddin, Nuovo Cimento C 23, 217 (2000).

[8] J. A. Lockwood and W. R. Webber, J.Geophys.Res.Space Phys. 89, A1 (1984) 17.

[9] M. Abbrescia et al., Nucl. Instrum. Methods Phys. Res. A 417, 16 (1998).

[10] Clem J.M., Dorman L.I., "Neutron monitors response function” Space Science Reviews 93, (2000), 335-359

[11] M. Abbrescia et al., Eur. Phys. J. Plus (2011) 126: 61.

[12] M. Abbrescia et al., Eur. Phys. J. Plus (2013) 128: 62 